\title{
Reação de cultivares de trigo à ferrugem da folha e mancha amarela e responsividade a fungicidas
}

\author{
Reaction of wheat cultivars to leaf rust and yellow spot and responsiveness to fungicides
}

\author{
Nédio Rodrigo Tormen ${ }^{\mathrm{I}}$ Giuvan Lenz ${ }^{\mathrm{II}}$ Simone Gripa Minuzzi ${ }^{\mathrm{II}}$ Juliano Daniel Uebel ${ }^{\mathrm{II}}$ \\ Heraldo Skrebsky Cezar ${ }^{\text {II }}$ Ricardo Silveiro Balardin ${ }^{\text {II }}$
}

\section{RESUMO}

\begin{abstract}
Com o objetivo de avaliar a reação de cultivares de trigo à ferrugem da folha, causada por Puccinia triticina, $e$ mancha amarela, causada por Drechslera tritici-repentis, $e$ sua responsividade ao controle químico com fungicidas, foi conduzido experimento de campo durante os invernos de 2009 e 2010, Itaara-RS. As cultivares avaliadas foram 'FCEP 51', 'FCEP 52', 'CD 114', 'FCEP Campo Real', 'FCEP Cristalino', 'FCEP Nova Era', 'OR Marfim', 'OR Abalone', 'OR Safira' $e$ 'OR Pampeano'; na safra 2010, a cultivar 'CD 114' foi substituída pela OR Quartzo. Os tratamentos avaliados foram: $P 1$ - testemunha sem aplicação de fungicida; P2 fempropimorfe (560g ha $a^{-1}$ de i.a.); P3 - azoxistrobina + ciproconazol $\left(60+24 \mathrm{~g} \mathrm{ha} \mathrm{a}^{-1}\right.$ de i.a.); e P4 - piraclostrobina + metconazol $\left(60+97,5 g\right.$ ha $a^{-1}$ de i.a.). Após a segunda aplicação, efetuaram-se avaliações semanais de severidade das doenças, cujos dados foram utilizados para o cálculo da Área Abaixo da Curva de Progresso da Doença (AACPD). Também foram determinados a produtividade e o peso do hectolitro. No que se refere à suscetibilidade às doenças, as cultivares estudadas apresentaram respostas distintas de um ano para o outro. Para ferrugem, o comportamento mostrou-se similar em ambos os anos, enquanto que, para mancha amarela, houve variação. Quanto à resposta ao controle químico, ocorreram variações de acordo com o patógeno e com o grau de suscetibilidade das cultivares. $O$ uso integrado de resistência genética e controle químico mostrou-se mais efetivo para ferrugem da folha.
\end{abstract}

Palavras-chave: cultivares de trigo, doenças, controle genético, controle químico.

\section{ABSTRACT}

Aiming to evaluate the susceptibility of wheat cultivars to leaf rust and yellow spot and their responsiveness to chemical fungicides, two field trials were carried out, during 2009 and 2010 winter's. The cultivars evaluated were: 'FCEP 51', 'FCEP 52', 'CD114', 'FCEP Campo Real', 'FCEP Cristalino', 'FCEP Nova Era', 'OR Marfim', 'OR Abalone', 'OR Safira' and 'OR Pampeano'. In 2010, the 'CD 114' cultivar was replaced by Quartzo. The treatments were: P1 - Without fungicide application; P2 - fenpropimorph (560g ha-l of a.i.); $P 3$ - azoxystrobin + cyproconazole $\left(60+24 g h a^{1}\right.$ of a.i.) and; P4 -

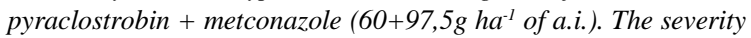
of leaf rust and tan spots were assessed weekly after the second spray and the data were utilized to calculate the Area Under the Disease Progress Curve (AUDPC). The grain yield and hectoliter weight were assessed at the harvest time. Regarding to susceptibility to diseases, the cultivars had different responses from one year to another. For leaf rust, the behavior was found to be similar in both studies, while for yellow spot was variation. Regarding to the chemical control, response variations were observed according to the pathogen and the susceptibility's degree of the cultivars. The integrated use of genetic resistance and chemical control was more effective for leaf rust.

Key words: wheat cultivars, diseases, genetic control, chemical control.

\section{INTRODUÇÃO}

A ocorrência de doenças da parte aérea na cultura do trigo pode causar reduções significativas na produtividade e na qualidade de grãos, explicando em parte as flutuações na produção desse cereal na região Sul do Brasil. A magnitude dos danos causados

'Departamento de Fitopatologia, Instituto de Ciências Biológicas, Universidade de Brasília (UNB), 70910-900, Brasília, DF, Brasil. E-mail: nedio.tormen@gmail.com. Autor para correspondência.

"Departamento de Defesa Fitossanitária, Centro de Ciências Rurais (CCR), Universidade Federal de Santa Maria (UFSM), Santa Maria, RS, Brasil. 
depende da suscetibilidade da cultivar, agressividade do patógeno, estádio de desenvolvimento da cultura no momento da infecção e das condições ambientais de cada ano e local (BARROS et al., 2006). Os danos tornam-se maiores à medida que duas ou mais doenças incidem de forma simultânea na lavoura.

Na região Sul do Brasil, destacam-se as doenças foliares, especialmente a ferrugem da folha e a mancha amarela. A ferrugem da folha do trigo, causada por Puccinia triticina, é uma doença importante em praticamente todas as regiões onde o trigo é cultivado no mundo (SMALE et al., 1998) e de ocorrência frequente no Sul do Brasil. A elevada frequência e o constante surgimento de novas raças do patógeno fazem da ferrugem da folha uma das doenças que mais limitam a produtividade do trigo no Brasil (REIS et al., 2006). A ferrugem da folha ocorre principalmente em cultivares suscetíveis e é pouco influenciada por práticas culturais (REIS \& CASA, 1997). A mancha amarela da folha do trigo, causada por Drechslera tritici-repentis, é de ocorrência mundial, sendo uma das principais doenças que causam perdas significativas à cultura na região Sul, onde o clima na primavera é bastante favorável ao seu desenvolvimento (SANTANA, 2009).

Para o controle de doenças na cultura do trigo, conforme preconiza o conceito de manejo integrado, todas as práticas disponíveis devem ser adotadas, visando a manter a população de organismos nocivos abaixo do limiar de dano econômico. Práticas tomadas isoladamente não se mostram tão eficientes, como quando usadas conjuntamente. Todavia, como as referidas doenças são causadas por patógenos de características diferentes, duas ferramentas - a resistência genética e o controle químico - são capazes de impactar significativamente ambas as doenças e minimizar os danos na lavoura.

O melhoramento genético de cultivares para resistência ou tolerância às doenças é, sem dúvida, o meio mais eficaz e econômico de reduzir os danos causados por elas. Porém, não existe no mercado uma única cultivar com resistência para todas as doenças foliares (REUNIÃO, 2008). Além disso, o estreitamento da base genética do germoplasma atualmente disponível e a plasticidade da população patogênica de algumas doenças têm ocasionado a quebra de resistência das cultivares e consequente necessidade de sua substituição (FELÍCIO et al., 2008). Da mesma forma, apesar de o controle químico apresentar boa eficiência no combate às doenças, a aplicação massiva de fungicidas pode gerar a seleção de raças dos patógenos que apresentem resistência aos ingredientes ativos utilizados, pondo em risco a eficiência do método e, muitas vezes, determinando a retirada de bons produtos do mercado (GHINI \& KIMATI, 2000).

Nesse contexto, quando resistência genética e controle químico são aliados, além de maior eficiência de controle das doenças, há maior preservação da eficácia de ambas. Sendo assim, o emprego de cultivares com algum nível de resistência genética, aliada à aplicação de fungicidas, é aspecto fundamental dentro de um programa de manejo integrado de doenças. $\mathrm{O}$ objetivo do presente trabalho foi avaliar a reação de dez cultivares de trigo à ferrugem da folha e à mancha amarela do trigo e a resposta delas a programas de controle com fungicidas.

\section{MATERIAL E MÉTODOS}

O experimento foi conduzido em campo durante os anos de 2009 e 2010, Itaara-RS. As cultivares foram semeadas no mesmo local nos dois anos, em sucessão à cultura da soja, utilizando espaçamento entre linhas de $0,17 \mathrm{~m}$ e uma população de 330 plantas $\mathrm{m}^{-2}$; a adubação de base utilizada foi de $150 \mathrm{~kg} \mathrm{ha}^{-1} \mathrm{da}$ fórmula 08-28-18. A semeadura foi realizada no período final da época recomendada para a região, buscando submeter as cultivares à maior pressão de inóculo, sendo realizada nos dias 27 de junho e 30 de julho, para os anos de 2009 e 2010, respectivamente. As demais práticas de manejo foram empregadas de acordo com as Indicações Técnicas da Comissão Sul-Brasileira de Pesquisa de Trigo (2009).

Os tratamentos foram dispostos no delineamento de blocos ao acaso com quatro repetições, em esquema fatorial $10 \times 4$, representado por dez cultivares de trigo e quatro programas de controle das doenças. No ano de 2009, utilizaram-se as cultivares 'FCEP 51', 'FCEP 52', 'CD 114', 'FCEP Campo Real', 'FCEP Cristalino', 'FCEP Nova Era', 'OR Marfim', 'OR Abalone', 'OR Safira' e 'OR Pampeano'; em 2010, devido à indisponibilidade de sementes, a cultivar ' $\mathrm{CD}$ 114' foi substituída pela 'OR Quartzo'. Os programas de controle empregados foram: P1 - testemunha sem aplicação de fungicida; $\mathrm{P} 2$ - fempropimorfe $\left(560 \mathrm{~g} \mathrm{ha}^{-1}\right.$ de i.a.); $\mathrm{P} 3$ - azoxistrobina + ciproconazol $\left(60+24 \mathrm{~g} \mathrm{ha}^{-1}\right.$ de i.a.); e P4 - piraclostrobina + metconazol (60+97,5g ha $^{-1}$ de i.a.). Em ambos os anos, todas as cultivares receberam duas aplicações de cada um dos tratamentos, exceto para o programa sem aplicação de fungicida. Em 2009, as aplicações foram realizadas entre os estádios fenológicos 7 e 10 para a primeira e 10 e 10.3 para a segunda aplicação (LARGE, 1954); em 2010, a aplicação dos tratamentos foi realizada entre os estádios 7 e 10 e 10.4 e 10.5.4, para a primeira e segunda aplicação, respectivamente. A aplicação dos fungicidas 
foi realizada com equipamento de pressão constante propelido por $\mathrm{CO}_{2}$, provido de barra de aplicação com quatro pontas de pulverização do tipo Teejet XR 11002 de jato plano de faixa ampliada, calibrado para vazão de $150 \mathrm{~L} \mathrm{ha}^{-1}$ e pressão constante de $270 \mathrm{kPa}$.

As variáveis mensuradas foram: severidade da ferrugem da folha e mancha amarela, produtividade de grãos e peso do hectolitro $(\mathrm{PH})$. A severidade das doenças foi estimada atribuindo-se notas da porcentagem de área foliar com sintomas das doenças para a folha bandeira, em 10 plantas por unidade experimental. A produtividade de grãos foi estimada pela colheita de todas as plantas da área útil $\left(8 \mathrm{~m}^{2}\right)$ de cada unidade experimental. A massa de grãos e a umidade de cada parcela foram determinadas, sendo posteriormente convertidas a $13 \%$ de umidade para o cálculo da produtividade. Com base nos dados de severidade das doenças, calculou-se a Área Abaixo da Curva de Progresso da Doença (AACPD) (CAMPBELL \& MADDEN, 1990). Os dados obtidos foram submetidos à análise de variância e as médias ao teste de comparação múltipla de médias de Tukey a 5\% de significância, utilizando o pacote estatístico Assistat (SILVA\&AZEVEDO, 2002).

\section{RESULTADOS}

A análise de variância dos dados apresentou valores de F significativos para cultivares, programas de controle e a interação entre esses dois fatores, em todos os parâmetros avaliados, nos dois anos de estudo. Verificaram-se diferenças significativas entre cultivares quanto à reação às doenças e também na responsividade delas aos programas de controle químico empregados. Em virtude da necessidade de substituição de uma das cultivares em 2010, cada experimento foi analisado de forma independente.

Para ferrugem da folha, observando os valores de AACPD, é possível identificar três grupos de cultivares quanto ao comportamento em relação à doença (Tabela 1). Algumas cultivares mostraram-se altamente suscetíveis, com AACPD muito acima da média (Nova Era, FCEP 51, FCEP 52), outras apresentaram suscetibilidade intermediária(CD114, Abalone, Cristalino e Safira) e ainda outras mostraram ser pouco suscetíveis à ferrugem da folha (Campo Real, Marfim e Pampeano). A cultivar 'Nova Era' foi a mais suscetível à ferrugem da folha e também a que apresentou maior redução na AACPD pelo emprego do controle químico, independente do programa de controle. Comparando a média dos dois melhores programas de controle (P3 e P4) com o programa testemunha, observou-se redução de $95,09 \%$ na AACPD nessa cultivar. A menor suscetibilidade à doença foi observada na cultivar 'Marfim', que não apresentou diferença estatística entre os diferentes programas de controle, não justificando assim a aplicação de fungicidas para o controle da ferrugem da folha.

Para mancha amarela, a diferença observada entre cultivares não foi tão pronunciada quanto para ferrugem da folha (Tabela 1). As cultivares 'CD 114', 'Cristalino', 'Marfim', 'Abalone' e 'Pampeano' não apresentaram diferenças significativas entre os diferentes programas de controle. As demais cultivares apresentaram redução na AACPD em pelo menos um dos programas de controle com aplicação de fungicida quando comparados ao programa sem controle. A cultivar 'FCEP 51' foi a mais suscetível à mancha amarela e também uma das que apresentou a maior redução na AACPD pelo emprego do controle químico entre as dez cultivares estudadas. A menor AACPD foi observada na cultivar 'Nova Era', apresentando um menor grau de suscetibilidade à doença. Para a produtividade de grãos, houve resposta positiva das cultivares ao controle químico, mas a magnitude da resposta variou bastante entre elas (Tabela 2). A amplitude do incremento na produtividade entre o pior e o melhor programa de controle variou de $236 \mathrm{~kg} \mathrm{ha}^{-1}$ ('FCEP Cristalino') a $748 \mathrm{~kg} \mathrm{ha}^{-1}$ ('FCEP 52'), respectivamente. $\mathrm{O}$ incremento médio na produtividade das cultivares pela aplicação de fungicida, considerando a média dos melhores programas de controle (P3 e P4), foi de $28 \%$.

Para o peso do hectolitro, somente houve diferença entre os programas de controle para as cultivares 'Campo Real' e 'Marfim', em que os programas P2 e P3 para a primeira e P3 e P4 para a segunda foram os melhores (Tabela 2). A cultivar 'Pampeano' apresentou o maior PH $(73,19)$, não diferindo das cultivares 'Cristalino' e 'Abalone'; o menor PH foi observado na cultivar 'FCEP 52' (62,52), não diferindo da 'CD 114'. Os valores relativamente baixos de PH observados nesse experimento se devem em parte ao atraso no processo de colheita, devido aos altos índices pluviométricos ocorridos durante o período de maturação das cultivares.

Em 2010, a reação das cultivares à ferrugem da folha foi bastante semelhante ao ano anterior, permitindo novamente agrupá-las com base na sua AACPD (Tabela 3). As cultivares 'Campo Real', 'Marfim', 'Cristalino' e 'Pampeano' foram pouco suscetíveis à ferrugem da folha; nessas cultivares, não houve diferença significativa na AACPD pelo emprego do controle químico. As cultivares 'FCEP 51', 'FCEP 52', 'Quartzo', 'Nova Era', 'Abalone' e 'Safira' mostraram-se bastante suscetíveis à doença e 
Tabela 1 - Área abaixo da curva de progresso de ferrugem da folha e mancha amarela, em dez cultivares de trigo, submetidas a programas de controle com fungicidas. Itaara, RS, 2009.

\begin{tabular}{|c|c|c|c|c|c|}
\hline Cultivar & $\mathrm{P} 1^{1}$ & $\mathrm{P} 2^{2}$ & $\mathrm{P}^{3}$ & $\mathrm{P} 4^{4}$ & Média \\
\hline 'FCEP 51' & $386,3 \mathrm{cA}^{5}$ & $149,0 \mathrm{cB}$ & $18,3 \mathrm{abC}$ & $23,3 \mathrm{aC}$ & $144,2 \mathrm{c}$ \\
\hline 'FCEP 52' & 564,3 bA & $263,5 \mathrm{bB}$ & $13,1 \mathrm{bcC}$ & $15,5 \mathrm{abC}$ & $214,1 \mathrm{~b}$ \\
\hline 'CD 114' & $104,3 \mathrm{eA}$ & $46,1 \mathrm{~dB}$ & $0,0 \mathrm{cC}$ & $0,0 \mathrm{bC}$ & $37,6 \mathrm{e}$ \\
\hline 'Campo Real' & $20,4 \mathrm{gA}$ & 11,1 efg $\mathrm{AB}$ & $0,0 \mathrm{cB}$ & $0,0 \mathrm{bB}$ & $8,4 \mathrm{gh}$ \\
\hline 'Cristalino’' & $45,2 \mathrm{fA}$ & 20,4 efB & $0,3 \mathrm{cC}$ & $0,3 \mathrm{bC}$ & $16,5 \mathrm{fg}$ \\
\hline 'Nova Era' & 644,7 aA & $333,0 \mathrm{aB}$ & $32,6 \mathrm{aC}$ & $30,6 \mathrm{aC}$ & $260,2 \mathrm{a}$ \\
\hline 'Marfim' & $6,4 \mathrm{gA}$ & $0,0 \mathrm{gA}$ & $0,0 \mathrm{cA}$ & $0,0 \mathrm{bA}$ & $1,6 \mathrm{~h}$ \\
\hline 'Abalone' & $124,2 \mathrm{dA}$ & $60,3 \mathrm{~dB}$ & $2,0 \mathrm{bcC}$ & $1,5 \mathrm{bC}$ & $47,0 \mathrm{~d}$ \\
\hline 'Safira' & 43,9 fA & 26,5 eB & $0,6 \mathrm{cC}$ & $0,8 \mathrm{bC}$ & $18,0 \mathrm{f}$ \\
\hline 'Pampeano' & $14,8 \mathrm{gA}$ & 7,2 fgAB & $0,0 \mathrm{cB}$ & $0,0 \mathrm{bB}$ & $5,5 \mathrm{~h}$ \\
\hline Média & $195,4 \mathrm{~A}$ & $91,7 \mathrm{~B}$ & $6,7 \mathrm{C}$ & $7,2 \mathrm{C}$ & 75,3 \\
\hline $\mathrm{CV} \%$ & & & 10,18 & & \\
\hline Cultivar & $\mathrm{P} 1$ & $\mathrm{P} 2$ & P3 & P4 & Média \\
\hline 'FCEP 51' & 104,6 aA & $82,3 \mathrm{aB}$ & $53,5 \mathrm{abC}$ & $52,3 \mathrm{abcC}$ & 73,2 a \\
\hline 'FCEP 52' & 73,5 bA & 61,3 bcdB & 56,3 abB & 49,5 bcB & $60,1 \mathrm{~b}$ \\
\hline 'CD 114' & $48,0 \mathrm{cA}$ & $50,6 \operatorname{def} A$ & $43,9 \mathrm{bcA}$ & $48,4 \mathrm{cA}$ & $47,7 \mathrm{c}$ \\
\hline 'Campo Real' & $75,1 \mathrm{bA}$ & $70,8 \mathrm{abAB}$ & $63,0 \mathrm{aB}$ & $64,1 \mathrm{abAB}$ & $68,3 \mathrm{a}$ \\
\hline 'Cristalino' & $41,1 \mathrm{cdA}$ & 41,5 efgA & 46,0 bA & $47,3 \mathrm{cA}$ & $44,0 \mathrm{c}$ \\
\hline 'Nova Era' & $35,5 \mathrm{cdA}$ & $18,9 \mathrm{hB}$ & $13,1 \mathrm{eB}$ & $15,4 \mathrm{~dB}$ & $20,7 \mathrm{e}$ \\
\hline ‘Marfim' & 73,4 bA & 68,0 abcA & $68,0 \mathrm{aA}$ & 64,9 aA & 68,6 a \\
\hline 'Abalone' & $27,6 \mathrm{dA}$ & $26,9 \mathrm{ghA}$ & $30,9 \mathrm{cdA}$ & $20,8 \mathrm{dA}$ & $26,5 \mathrm{de}$ \\
\hline 'Safira' & $39,6 \mathrm{cdA}$ & 38,9 fgA & $29,0 \mathrm{dAB}$ & $24,5 \mathrm{~dB}$ & $33,0 \mathrm{~d}$ \\
\hline 'Pampeano' & 64,5 bA & $55,8 \mathrm{cdeA}$ & 56,2 abA & $53,5 \mathrm{abcA}$ & $57,5 \mathrm{~b}$ \\
\hline Média & $58,3 \mathrm{~A}$ & $51,5 \mathrm{~B}$ & $46,0 \mathrm{C}$ & $44,1 \mathrm{C}$ & 50,0 \\
\hline CV \% & & & 13,0 & & \\
\hline
\end{tabular}

${ }^{1} \mathrm{P} 1$ - testemunha sem controle; ${ }^{2} \mathrm{P} 2$ - fempropimorfe $\left(560 \mathrm{~g}\right.$ ha ${ }^{-1}$ de i.a. $) ;{ }^{3} \mathrm{P} 3$ - azoxistrobina + ciproconazole $\left(60+24 \mathrm{~g} . h a^{-1}\right.$ de i.a.) e ${ }^{4} \mathrm{P} 4$ piraclostrobina + metconazole $\left(97,5+60 \mathrm{~g}\right.$.ha ${ }^{-1}$ de i.a.). ${ }^{5}$ Valores seguidos pela mesma letra minúscula na coluna e maiúscula na linha não diferem estatisticamente pelo teste de Tukey a $5 \%$ de probabilidade de erro. *Foram realizadas duas aplicações de cada programa de controle em todas as cultivares.

responsivas ao controle químico, com destaque para os programas de controle $\mathrm{P} 3$ e $\mathrm{P} 4$, que proporcionaram eficácia de controle próxima a 100\%. As cultivares 'FCEP 51' e 'Marfim' apresentaram a maior e menor suscetibilidade à doença, respectivamente.

Os dados de AACPD da mancha amarela novamente mostraram haver menor variabilidade entre cultivares quanto à reação à doença e, contrariamente ao observado para ferrugem da folha, não houve consonância no comportamento das cultivares de um ano para outro (Tabela 3). Destacaram-se como menos suscetíveis as cultivares 'Campo Real' e 'Abalone' e, como mais suscetíveis, 'FCEP 52', 'Quartzo', 'Marfim' e 'Safira'. A redução na AACPD em relação à testemunha sem controle, considerando a média dos programas $\mathrm{P} 2, \mathrm{P} 3$ e $\mathrm{P} 4$ foi de $64 \%$; não houve diferença estatística entre os programas com aplicação de fungicidas, exceto para as cultivares 'Safira' e 'Nova Era', para as quais o programa $\mathrm{P} 2$ para a primeira e os programas $\mathrm{P} 3$ e $\mathrm{P} 4$ para a segunda foram os melhores.

A cultivar que apresentou maior incremento em produtividade pela aplicação de fungicidas, a exemplo do ano anterior, foi a 'FCEP 52', a qual produziu $71 \%$ a mais no melhor tratamento, quando comparado com programa sem fungicida (Tabela 4 ). O menor incremento foi observado na cultivar 'Marfim' $(6,6 \%) \mathrm{e}$ nas cultivares 'Campo Real', 'Nova Era' e 'Pampeano' não houve diferença entre os programas de controle, não justificando assim o emprego do controle químico.

Quanto ao $\mathrm{PH}$, não houve diferença significativa entre os programas de controle somente para as cultivares 'Campo Real', 'Cristalino' e 
Tabela 2 - Produtividade de grãos e peso do hectolitro em dez cultivares de trigo, submetidas a quatro programas de controle químico de ferrugem da folha e mancha amarela. Itaara, RS, 2009.

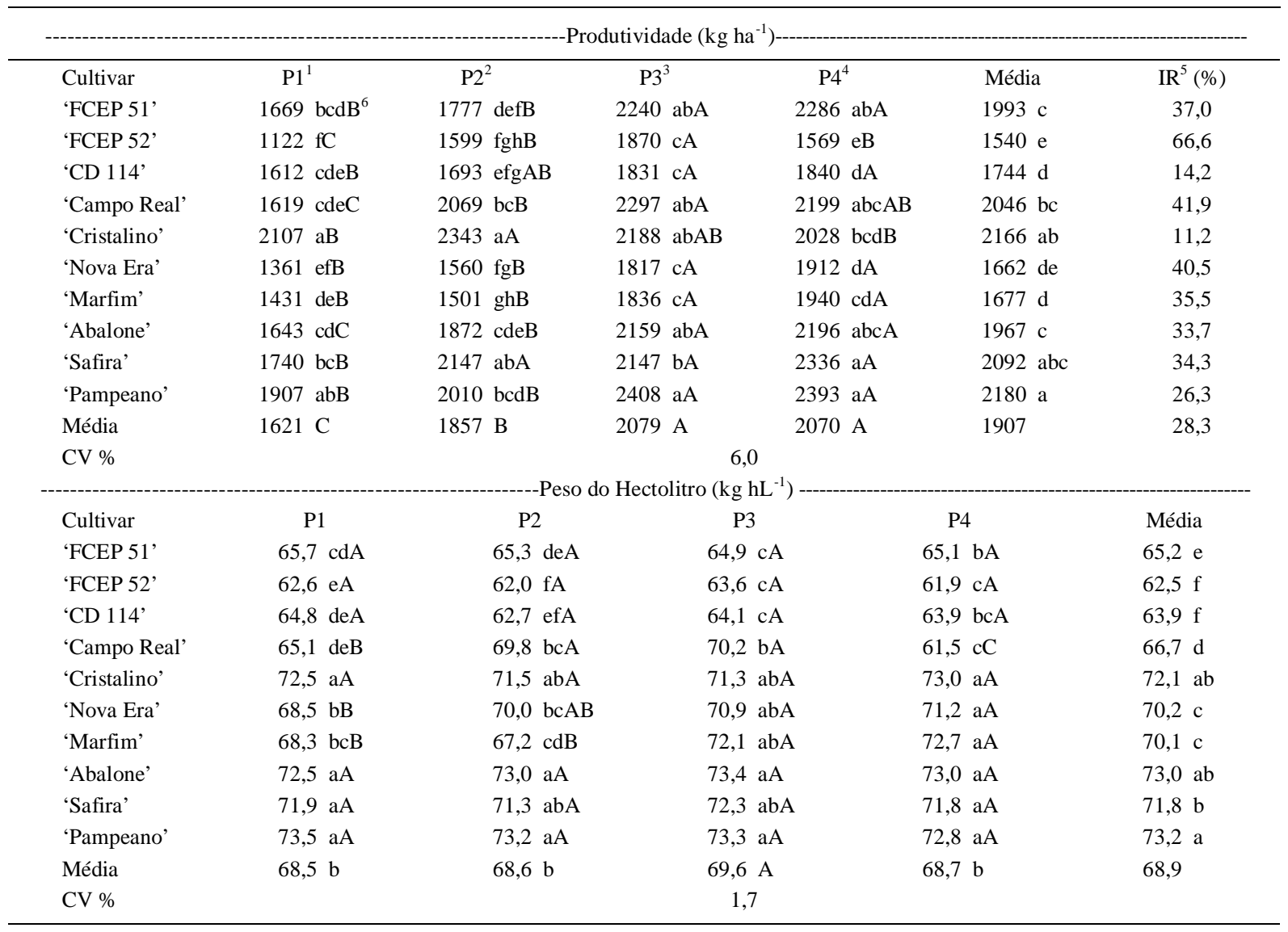

${ }^{1} \mathrm{P} 1$ - testemunha sem controle; ${ }^{2} \mathrm{P} 2$ - fempropimorfe $\left(560 \mathrm{~g} \mathrm{ha}{ }^{-1}\right.$ de i.a. $) ;{ }^{3} \mathrm{P} 3$ - azoxistrobina + ciproconazole $\left(60+24 \mathrm{~g} . h a^{-1}\right.$ de i.a. $)$ e ${ }^{4} \mathrm{P} 4$ piraclostrobina + metconazole $\left(97,5+60 \mathrm{~g} . \mathrm{ha}^{-1}\right.$ de i.a.). ${ }^{5} \mathrm{IR}$ - incremento relativo em produtividade do melhor programa de controle em relação à testemunha sem fungicida. ${ }^{6}$ Valores seguidos pela mesma letra minúscula na coluna e maiúscula na linha não diferem estatisticamente pelo teste de Tukey a 5\% de probabilidade de erro. *Foram realizadas duas aplicações de cada programa de controle em todas as cultivares.

'Pampeano' (Tabela 4). Nas demais cultivares, destacouse o programa $\mathrm{P} 4$, que apresentou os valores mais elevados de PH nas cultivares 'FCEP 51', 'FCEP 52', 'Quartzo', 'Nova Era', 'Abalone', 'Safira' e também na média de todas as cultivares.

\section{DISCUSSÃO}

Analisando os dados de AACPD da mancha amarela e ferrugem da folha, verificou-se menor eficácia dos fungicidas no controle da primeira. Em razão da base genética encontrada nos sistemas patógenohospedeiro, verifica-se um maior nível de resistência aos fungos biotróficos do que aos necrotróficos. $\mathrm{O}$ uso de cultivares resistentes é a medida preferencial de controle dos parasitas biotróficos, pois esses patógenos não são controláveis pela rotação de culturas como ocorre com os necrotróficos (REIS \& CASA, 2007). Na média das cultivares, comparando os dados do programa sem fungicida com a média dos três programas com fungicida, há redução de $84,3 \%$ para ferrugem da folha e $17,4 \%$ para a mancha amarela. Esses resultados se devem provavelmente às características inerentes às doenças, mecanismos de resistência que atuam no hospedeiro e também ao modo de ação dos fungicidas utilizados. O progresso de uma doença pode ser explicado pelo aparecimento de novas lesões e/ou pelo crescimento daquelas já existentes. A taxa de expansão da lesão é um componente importante no processo epidêmico de manchas (MENEGON et al., 2005), diferentemente da ferrugem, em que o aparecimento de novas lesões é mais importante. Essa 
condição, associada à habilidade de as plantas de trigo em suprimir ou retardar, em algum grau de intensidade, a atividade dos patógenos, pode explicar parte da variação observada na resposta ao controle químico entre as doenças. Os dois programas de controle mais eficazes para mancha amarela e ferrugem da folha são compostos pela mistura de um triazol com uma estrobilurina. Os fungicidas do grupo dos triazóis atuam na síntese de esteróis e possuem efeito curativo marcante, ao passo que as estrobilurinas atuam na respiração mitocondrial e seu efeito maior é na germinação de esporos (REIS et al., 2001).

$\mathrm{O}$ incremento médio em produtividade das cultivares no melhor programa de controle foi de 28,3 e $25,8 \%$ nos anos de 2009 e 2010, respectivamente. Esses valores são inferiores aos encontrados por BARROS et al. (2006), que, trabalhando com seis cultivares de trigo, encontraram incremento médio de 39,17\%. A não existência de uma relação singular entre AACPD das doenças avaliadas e a produtividade sinaliza para o fato de que há fatores genéticos atuando, seja pela rusticidade ou mesmo tolerância de cada cultivar quando submetida à alta pressão de doença. Nesse sentido, há cultivares que mesmo apresentando baixos valores de AACPD sofrem grandes perdas em produtividade na ausência de controle químico, como ocorreu com a cultivar 'Campo Real' nos dois anos de estudo. Existem ainda cultivares que são extremamente responsivas ao controle químico, como a 'FCEP 52', em que a diferença em produtividade da testemunha

Tabela 3 - Área abaixo da curva de progresso da ferrugem da folha e mancha amarela em dez cultivares de trigo, submetidas a quatro programas de controle das doenças. Itaara, RS, 2010.

\begin{tabular}{|c|c|c|c|c|c|}
\hline Cultivar & $\mathrm{P} 1^{1}$ & $\mathrm{P} 2^{2}$ & $\mathrm{P} 3^{3}$ & $\mathrm{P} 4^{4}$ & Média \\
\hline 'FCEP 51' & $835,6 \mathrm{aA}^{5}$ & $70,0 \mathrm{bB}$ & $8,1 \mathrm{aC}$ & $8,1 \mathrm{aC}$ & 230,5 a \\
\hline 'FCEP 52' & 483,0 bA & $112,9 \mathrm{aB}$ & $6,8 \mathrm{aC}$ & $7,9 \mathrm{aC}$ & $152,6 \mathrm{~b}$ \\
\hline ‘Quartzo’ & $476,9 \mathrm{bA}$ & $26,7 \mathrm{cdB}$ & $5,7 \mathrm{aB}$ & $5,0 \mathrm{aB}$ & $128,6 \mathrm{c}$ \\
\hline 'Campo Real' & $15,1 \mathrm{dA}$ & $1,9 \mathrm{dA}$ & $0,0 \mathrm{aA}$ & $0,0 \mathrm{aA}$ & $4,3 \mathrm{e}$ \\
\hline 'Cristalino' & $28,4 \mathrm{dA}$ & $8,2 \mathrm{dA}$ & $0,8 \mathrm{aA}$ & $2,5 \mathrm{aA}$ & $10,0 \mathrm{e}$ \\
\hline 'Nova Era' & 503,1 bA & $51,2 \mathrm{bcB}$ & $6,3 \mathrm{aC}$ & $2,6 \mathrm{aC}$ & $140,8 \mathrm{bc}$ \\
\hline 'Marfim' & $2,2 \mathrm{dA}$ & $0,0 \mathrm{dA}$ & $0,0 \mathrm{aA}$ & $0,0 \mathrm{aA}$ & $0,5 \mathrm{e}$ \\
\hline 'Abalone' & $481,3 \mathrm{bA}$ & 30,6 bcdB & $6,3 \mathrm{aB}$ & $5,8 \mathrm{aB}$ & $131,0 \mathrm{c}$ \\
\hline 'Safira' & $318,5 \mathrm{cA}$ & $20,3 \mathrm{cdB}$ & $2,5 \mathrm{aB}$ & $1,1 \mathrm{aB}$ & $85,6 \mathrm{~d}$ \\
\hline 'Pampeano' & $6,9 \mathrm{dA}$ & $1,5 \mathrm{dA}$ & $0,0 \mathrm{aA}$ & $0,1 \mathrm{aA}$ & $2,1 \mathrm{e}$ \\
\hline Média & $315,1 \mathrm{~A}$ & $32,3 \mathrm{~b}$ & $3,7 \mathrm{c}$ & $3,3 \mathrm{c}$ & 88,6 \\
\hline $\mathrm{CV} \%$ & & & 20,46 & & \\
\hline Cultivar & $\mathrm{P} 1$ & $\mathrm{P} 2$ & $\mathrm{P} 3$ & P4 & Média \\
\hline 'FCEP 51' & $77,0 \mathrm{bA}$ & $21,9 \mathrm{deB}$ & $18,6 \mathrm{bcB}$ & $17,5 \mathrm{bcB}$ & 33,8 cde \\
\hline 'FCEP 52' & $136,5 \mathrm{aA}$ & 38,5 bcdB & 35,0 bB & $29,3 \mathrm{bcB}$ & $59,8 \mathrm{~b}$ \\
\hline 'Quartzo’' & $122,1 \mathrm{aA}$ & 35,2 bcdB & $35,2 \mathrm{bB}$ & 30,8 bB & $55,8 \mathrm{~b}$ \\
\hline 'Campo Real' & $48,7 \mathrm{dA}$ & 29,1 bcdeB & $27,0 \mathrm{bcB}$ & $17,4 \mathrm{bcB}$ & 30,6 de \\
\hline 'Cristalino’' & 74,8 bA & 39,4 abcB & $27,6 \mathrm{bcB}$ & $26,3 \mathrm{bcB}$ & $42,0 \mathrm{c}$ \\
\hline 'Nova Era' & $68,3 \mathrm{bcA}$ & 35,4 bcdB & $17,5 \mathrm{cC}$ & $17,7 \mathrm{bcC}$ & $34,7 \mathrm{~cd}$ \\
\hline ‘Marfim’ & $129,5 \mathrm{aA}$ & $56,0 \mathrm{aB}$ & $56,4 \mathrm{aB}$ & $50,8 \mathrm{aB}$ & 73,2 a \\
\hline 'Abalone' & $54,3 \mathrm{cdA}$ & $15,6 \mathrm{eB}$ & $18,6 \mathrm{bcB}$ & $13,0 \mathrm{cB}$ & $25,3 \mathrm{e}$ \\
\hline 'Safira' & $129,5 \mathrm{aA}$ & $43,8 \mathrm{abC}$ & $67,4 \mathrm{aB}$ & $66,1 \mathrm{aB}$ & $76,7 \mathrm{a}$ \\
\hline 'Pampeano' & $72,6 \mathrm{bA}$ & $23,8 \mathrm{cdeB}$ & $35,0 \mathrm{bB}$ & $27,8 \mathrm{bcB}$ & $39,8 \mathrm{c}$ \\
\hline Média & $91,3 \mathrm{~A}$ & $33,9 \mathrm{~b}$ & $33,8 \mathrm{~b}$ & $29,7 \mathrm{~b}$ & 47,2 \\
\hline $\mathrm{CV} \%$ & & & 15,95 & & \\
\hline
\end{tabular}

${ }^{1} \mathrm{P} 1$ - testemunha sem controle; ${ }^{2} \mathrm{P} 2$ - fempropimorfe $\left(560\right.$ g.ha ${ }^{-1}$ de i.a. $) ;{ }^{3} \mathrm{P} 3$ - azoxistrobina + ciproconazole $\left(60+24\right.$ g.ha ${ }^{-1}$ de i.a. $)$ e ${ }^{4} \mathrm{P} 4$ piraclostrobina + metconazole $\left(97,5+60\right.$ g.ha ${ }^{-1}$ de i.a.). ${ }^{5}$ Valores seguidos pela mesma letra minúscula na coluna e maiúscula na linha não diferem estatisticamente pelo teste de Tukey a $5 \%$ de probabilidade de erro. *Foram realizadas duas aplicações de cada programa de controle em todas as cultivares.

Ciência Rural, v.43, n.2, fev, 2013. 
Tabela 4 - Produtividade de grãos e peso do hectolitro em dez cultivares de trigo submetidas a quatro programas de controle químico de Puccinia triticina e Drechslera tritici-repentis. Itaara, RS, 2010.

\begin{tabular}{|c|c|c|c|c|c|c|}
\hline Cultivar & $\mathrm{P} 1^{1}$ & $\mathrm{P} 2^{2}$ & $\mathrm{P} 3^{3}$ & $\mathrm{P} 4^{4}$ & Média & $\operatorname{IR}^{5}(\%)$ \\
\hline 'FCEP 51' & $1725 \mathrm{bcC}^{6}$ & $1948 \mathrm{bBC}$ & 2350 abcA & $2273 \mathrm{bcAB}$ & $2074 \mathrm{~b}$ & 36,2 \\
\hline 'FCEP 52' & $1418 \mathrm{cB}$ & $1573 \mathrm{bcdB}$ & $2425 \mathrm{abA}$ & 2408 bA & $1956 \mathrm{bc}$ & 71,1 \\
\hline ‘Quatzo’ & $2278 \mathrm{aB}$ & $2500 \mathrm{aB}$ & $2575 \mathrm{aAB}$ & $2875 \mathrm{aA}$ & $2557 \mathrm{a}$ & 13,1 \\
\hline 'Campo Real' & $1675 \mathrm{bcA}$ & $1975 \mathrm{bA}$ & $1998 \mathrm{bcA}$ & $1915 \mathrm{cdA}$ & $1891 \mathrm{bcd}$ & 19,3 \\
\hline 'Cristalino' & $2000 \mathrm{abB}$ & $2725 \mathrm{aA}$ & $2600 \mathrm{aA}$ & $2715 \mathrm{abA}$ & $2510 \mathrm{a}$ & 30,0 \\
\hline 'Nova Era' & $958 \mathrm{dA}$ & $1128 \mathrm{dA}$ & $1280 \mathrm{dA}$ & $1128 \mathrm{eA}$ & 1123 e & 33,7 \\
\hline 'Marfim' & $1283 \mathrm{cdA}$ & $1383 \mathrm{cdA}$ & $1368 \mathrm{dA}$ & $1163 \mathrm{eA}$ & 1299 e & 6,6 \\
\hline 'Abalone' & $1463 \mathrm{cB}$ & $1675 \mathrm{bcAB}$ & $1930 \mathrm{cA}$ & $1775 \mathrm{dAB}$ & $1711 \mathrm{~d}$ & 32,0 \\
\hline 'Safira' & $1525 \mathrm{cB}$ & $1725 \mathrm{bcAB}$ & $2033 \mathrm{bcA}$ & $1948 \mathrm{cdA}$ & $1808 \mathrm{~cd}$ & 33,3 \\
\hline 'Pampeano' & $2343 \mathrm{aA}$ & 2509 aA & $2598 \mathrm{aA}$ & $2564 \mathrm{abA}$ & $2504 \mathrm{a}$ & 10,9 \\
\hline Média & $1667 \mathrm{c}$ & $1914 \mathrm{~b}$ & $2116 a$ & 2076 a & 1943 & 26,9 \\
\hline CV \% & \multicolumn{6}{|c|}{10,19} \\
\hline \multicolumn{7}{|c|}{----Peso do Hectolitro (kg hL } \\
\hline Cultivar & $\mathrm{P} 1$ & $\mathrm{P} 2$ & P3 & P4 & & Média \\
\hline 'FCEP 51' & $75,7 \mathrm{aB}$ & 76,5 abcAB & 78,5 aA & $78,9 \mathrm{abA}$ & & 77,4 a \\
\hline 'FCEP 52' & 73,8 abcA & $70,6 \mathrm{~dB}$ & $74,0 \mathrm{cA}$ & $74,6 \mathrm{cdA}$ & & $73,3 \mathrm{e}$ \\
\hline 'Quatzo’' & $71,7 \mathrm{bcB}$ & $74,1 \mathrm{cAB}$ & $73,8 \mathrm{cAB}$ & $74,7 \mathrm{cdA}$ & & 73,6 de \\
\hline 'Campo Real' & 75,6 aA & 76,6 abcA & $75,2 \mathrm{bcA}$ & $75,7 \mathrm{cdA}$ & & $75,8 \mathrm{~b}$ \\
\hline 'Cristalino' & 76,1 aA & $73,7 \mathrm{cdA}$ & 75,6 abcA & $74,2 \mathrm{cdA}$ & & 74,9 bcd \\
\hline 'Nova Era' & $76,1 \mathrm{aB}$ & $78,2 \mathrm{aAB}$ & $78,0 \mathrm{abAB}$ & 79,7 aA & & 78,0 a \\
\hline ‘Marfim’ & $71,5 \mathrm{cB}$ & $74,7 \mathrm{bcA}$ & $75,2 \mathrm{bcA}$ & $72,9 \mathrm{dAB}$ & & 73,6 cde \\
\hline 'Abalone' & 74,5 abcB & 77,8 abA & 77,7 abA & 79,8 aA & & 77,4 a \\
\hline 'Safira' & $74,8 \mathrm{abAB}$ & $73,8 \mathrm{cB}$ & 75,6 abcAB & $76,5 \mathrm{bcA}$ & & $75,1 \mathrm{bc}$ \\
\hline 'Pampeano' & 74,5 abcA & 75,5 abcA & $75,3 \mathrm{bcA}$ & $74,7 \mathrm{cdA}$ & & 75,0 bcd \\
\hline Média & $74,4 \mathrm{c}$ & $75,1 \mathrm{bc}$ & $75,9 \mathrm{ab}$ & 76,2 a & & 75,4 \\
\hline CV \% & & & 1,80 & & & \\
\hline
\end{tabular}

${ }^{1} \mathrm{P} 1$ - testemunha sem controle; ${ }^{2} \mathrm{P} 2$ - fempropimorfe $\left(560\right.$ g.ha ${ }^{-1}$ de i.a. $) ;{ }^{3} \mathrm{P} 3$ - azoxistrobina + ciproconazole $\left(60+24\right.$ g.ha ${ }^{-1}$ de i.a. $)$ e ${ }^{4} \mathrm{P} 4$ piraclostrobina + metconazole $\left(97,5+60\right.$ g.ha ${ }^{-1}$ de i.a. $) .{ }^{5} \mathrm{IR}$ - incremento relativo em produtividade do melhor programa de controle em relação à testemunha sem fungicida. ${ }^{6}$ Valores seguidos pela mesma letra minúscula na coluna e maiúscula na linha não diferem estatisticamente pelo teste de Tukey a $5 \%$ de probabilidade de erro. *Foram realizadas duas aplicações de cada programa de controle em todas as cultivares.

para o melhor programa de controle foi de 747,5 e $1007,5 \mathrm{~kg} \mathrm{ha}^{-1}$, nos anos de $2009 \mathrm{e} 2010$, respectivamente. Por outro lado, há cultivares mais estáveis em relação à reação às doenças e também em produtividade, como observado na cultivar 'Pampeano', mostrando grande habilidade em suprimir e/ou retardar a atividade de ambos os patógenos. Além disso, a interação existente entre a expressão gênica e o ambiente pode resultar em modificações no comportamento de uma cultivar de um ano para outro, como resultado de variações ambientais. Essa pode ser a explicação para o comportamento da cultivar 'Marfim', que, mesmo apresentando baixa severidade de doenças nos dois anos, apresentou um incremento em produtividade excelente em 2009 e insignificante em 2010, pelo emprego do controle químico.

\section{CONCLUSÃO}

A sensibilidade das cultivares à mancha amarela variou de uma safra para outra, enquanto que, para ferrugem, o comportamento manteve-se estável.

As cultivares estudadas apresentaram diferentes níveis de suscetibilidade à ferrugem da folha e mancha amarela, diferindo quanto a sua responsividade ao controle químico.

A combinação de estratégias de manejo com uso de controle químico e genético teve maior efeito sobre ferrugem da folha do que sobre mancha amarela.

\section{REFERÊNCIAS}

BARROS B.C. et al. Resposta de cultivares de trigo (Triticum aestivum L.) ao controle químico das principais doenças fúngicas 
da cultura. Summa Phytopathologica, v.32, p.239-246, 2006. Disponível em: <http://www.scielo.br/scielo.php?pid=S0100$54052006000300005 \&$ script=sci_arttext $>$. Acesso em: 13 ago. 2012. doi: $10.1590 / \mathrm{S} 0100-54052006000300005$.

CAMPBELL, C.L.; MADDEN, L.V. Monitoring epidemics: diseases. In: -__- Introduction to plant disease epidemiology. New York: J. Wiley, 1990. Cap.6, p.107-128.

LARGE, E.C. Growth stages in cereal: illustration of the Feekes scale. Plant Pathology, v.3, p.129, 1954.

FELÍCIO, J.C. et al. Resistência à ferrugem da folha e potencial produtivo em genótipos de trigo. Bragantia, v.67, n.4, p.865863, 2008. Disponível em: <http://www.scielo.br/scielo.php? script $=$ sci_arttext $\&$ pid $=$ S0006-87052008000400007\& lng=pt\&nrm=iso>. Acesso em: 13 ago. 2012. doi: 10.1590/ S0006-87052008000400007.

GHINI, R.; KIMATI, H. Resistência de fungos a fungicidas. Jaguariúna: Embrapa Meio Ambiente, 2000. 78p.

MENEGON, A.P. et al. Expansão de lesão por manchas foliares em cevada e sua interação com a aplicação foliar de fungicidas. Fitopatologia Brasileira, v.30, p.134-138, 2005. Disponível em: <http://www.scielo.br/scielo.php?script=sci_arttext $\&$ pid $=$ S0100-41582005000200005\&lng=pt\&nrm=iso $>$. Acesso em: 13 ago. 2012. doi: 10.1590/S0100-41582005000200005.

SANTANA, F.M. Avaliação da eficiência de controle de mancha amarela em duas linhagens de trigo da Embrapa Trigo. Passo Fundo: Embrapa Trigo, 2009. 12p.

REIS, E.M.; CASA, R.T. Cereais de inverno. In: et al. Controle de doenças de plantas: grandes culturas. Brasília, DF: Ministério da Agricultura e do Abastecimento, 1997. V.2, p.231-289.
REIS, E.M.; CASA, R.T. Danos causados por fungos associados a sementes de cereais de inverno. Summa Phytopathologica, v.31, p.138-140, 2005.

REIS, E.M.; CASA, R.T. Doenças dos cereais de inverno: diagnose, epidemiologia e controle. 2.ed. Lages: Graphel, 2007. 176p.

REIS, E.M. et al. Manual de fungicidas: guia para o controle químico de doenças de plantas. 4.ed. Florianópolis: Insular, 2001. 176p. : il.

REIS, E.M. et al. Relações entre intensidade da ferrugem da folha, refletância da radiação solar e rendimento de grãos no patossistema ferrugem da folha de trigo Embrapa 16. Fitopatologia Brasileira, v.31, p.447-454, 2006. Disponível em: <http://www.scielo.br/scielo.php?script=sci_arttext\& $\mathrm{pid}=\mathrm{S} 0100-41582006000500003 \& \operatorname{lng}=\mathrm{pt} \& \mathrm{nrm}=\mathrm{iso}>$. Acesso em: 13 ago. 2012. doi: 10.1590/S0100-41582006000500003.

REUNIÃO DA COMISSÃO BRASILEIRA DE PESQUISA DE TRIGO E TRITICALE, Passo Fundo, RS. Informações técnicas para a safra 2009: trigo e triticale. Passo Fundo: Comissão Brasileira de Pesquisa de Trigo, 2008. 172p.

SILVA, F.A.S.; AZEVEDO, C.A.V. Versão do programa computacional Assistat para o sistema operacional Windows. Revista Brasileira de Produtos Agroindustriais, v.4, n.1, p.71-78, 2002. Disponível em: <http://www.deag.ufcg.edu.br/ rbpa/rev41/Art410.pdf>. Acesso em: 13 ago. 2012.

SMALE, M. et al. Estimating the economic impact of breeding nonspecific resistance to leaf rust in modern bread wheats. Plant Disease, v.82, p.1055-1061, 1998. Disponível em: <http://apsjournals.apsnet.org/doi/abs/10.1094/ PDIS.1998.82.9.1055>. Acesso em: 13 ago. 2012. doi: 10.1094/PDIS.1998.82.9.1055. 